\title{
COMPUTATIONAL ALGORITHM FOR THE EXPONENTIAL INTEGRAL
}

\section{A. SHARAF ${ }^{1}$ AND F. A. ALRAWJIH ${ }^{2}$}

\author{
${ }^{1}$ Department of Astronomy, Faculty of Science, King Abdulaziz University, Jeddah, Saudi Arabia \\ ${ }^{2}$ Department of Mathematics, Faculty of Science, University of Dammam, Dammam, Saudi Arabia
}

\section{ABSTRACT}

In this paper, an efficient computational algorithm for the exponential integral $E_{v}(z)$ was established for all $v=q_{1}+i q_{2}$ and $z=a+i b$. The algorithm based on the evaluation of the continued fraction expansion of $E_{v}(z)$. Numerical applications of the algorithm are also given together with $3 D$ plotes.

Keywords: Exponential integral, computational algorithm, Numerical applications.

\section{INTRODUCTION}

Beyond hydrological applications, the exponential integral involving complex exponentials is very widespread in mathematical physics as electromagnetism [1], and is to be found in standard texts dealing with functions arising in applied mathematics $[2,3,4]$. Also many algorithms have been created for the rapid and accurate calculation [5], novel approximation for the exponential integral function [6].

\section{Basic Formulations}

In fact, continued fraction expansions are generally more efficient tools for evaluating the classical functions than the more infinite power series. Their convergence is typically faster and more extensive than the series.

The continued fraction expansion of the exponential integral can be written as [7]

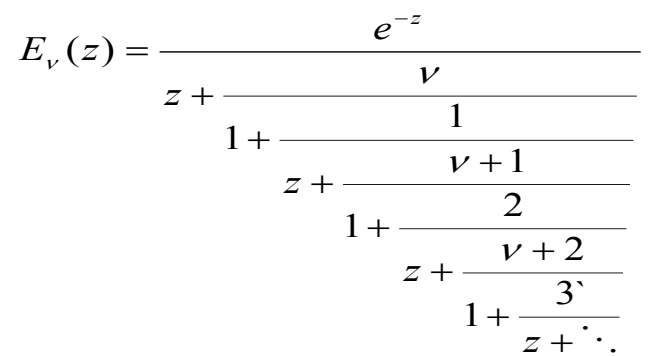

valid for all $v=q_{1}+i q_{2}$ and $z=a+i b$.

There are several methods available for the evaluation of continued fraction. Traditionally, the fraction was either computed from the bottom up, or the numerator and denominator of the $\mathrm{n}^{\text {th }}$ convergent were accumulated separately with three-term recurrence formulae. The draw back of the first method is, obviously, having to de- cide far down the fraction to being in order to ensure convergence. The draw back to the second method is that the numerator and denominator rapidly overflow numerically even though their ratio tends to a well defined limit. Thus, it is clear that an algorithm that works from top down while avoiding numerical difficulties would be ideal from a programming standpoint.

Gautschi [8] proposed very concise algorithm to evaluate continued fraction from the top down and may be summarized as follows. If the continued fraction is written as

$$
\mathrm{c}=\frac{\mathrm{n}_{1}}{\mathrm{~d}_{1}+\frac{\mathrm{n}_{2}}{\mathrm{~d}_{2}+\frac{\mathrm{n}_{3}}{\mathrm{~d}_{3}+\ddots}},}
$$

then initialize the following parameters

$\mathrm{a}_{1}=1, \quad \mathrm{~b}_{1}=\mathrm{n}_{1} / \mathrm{d}_{1}, \quad \mathrm{c}_{1}=\mathrm{n}_{1} / \mathrm{d}_{1}$,

and iterate $(k=1,2, \ldots)$ according to

$$
\begin{aligned}
\mathrm{a}_{\mathrm{k}+1} & =\frac{1}{1+\left[\frac{\mathrm{n}_{\mathrm{k}+1}}{\mathrm{~d}_{\mathrm{k}} \mathrm{d}_{\mathrm{k}+1}}\right] \mathrm{a}_{\mathrm{k}},}, \\
\mathrm{b}_{\mathrm{k}+1} & =\left[\mathrm{a}_{\mathrm{k}+1}-1\right] \mathrm{b}_{\mathrm{k}} \\
\mathrm{c}_{\mathrm{k}+1} & =\mathrm{c}_{\mathrm{k}}+\mathrm{b}_{\mathrm{k}+1} .
\end{aligned}
$$

In the limit, the $c$ sequence converges to the value of the continued fraction.

Continued fraction method was used in many problems in astrophysics [e.g. $9 \& 10$ ] as well as in the special functions of astrodynamics [e.g. $11 \& 12]$ 


\section{Computational developments}

\subsection{Analytical expressions of the n's and d's functions}

In order to apply Gautschi's algorithm, we have to set up the first analytical expressions of the $n$ 's and $d$ 's functions, which we derived as

$$
\begin{aligned}
& n_{1}=e^{-z} ; \\
& n_{i}=\left\{\begin{array}{lr}
{[i / 2]} & \text { f i odd, } \\
v+[i / 2]-1 & \mathbf{f} \text { i even. }
\end{array} \quad i \geq 2,\right. \\
& d_{j}=\left\{\begin{array}{ll}
\mathrm{z} & \mathbf{f} \text { jodd, } \\
1 & \mathbf{f} \text { j even, }
\end{array} \mathrm{j} \geq 1 ;\right.
\end{aligned}
$$

where $[x]$ is the integer part of $x$ less or equal to $x$.

\subsection{Examples}

Applying Gautschi's algorithm with the above values of the $n$ 's and $d$ 's functions for the values of $v \& z$ listed in the first two columns of Table (1), we get for $E_{v}(z)$ the values listed in the third column of the table.

\subsection{D-Representations}

Figure (1) shows 3D representations of $E_{-v}(x+\dot{y}) ; \quad v=0(\emptyset) 5 \quad \forall x \in[-1,1]$ and $y \in[-1,1]$.

Table (1): Values of $E_{\nu}(z)$ for some $v$ and $z$.

\begin{tabular}{|c|c|c|}
\hline$v$ & $z$ & $E_{v}(z)$ \\
\hline 4 & 6 & 0.0002570434 \\
\hline 0 & 3 & 0.0165956895 \\
\hline-2.2 & 3.56 & 0.0146679470 \\
\hline $7+2 \mathrm{i}$ & 5 & $0.0004695927-0.0007072864 \mathrm{i}$ \\
\hline$-2 \mathrm{i}$ & $-4 \mathrm{i}$ & $0.1331510002-0.1031333760 \mathrm{i}$ \\
\hline$-2 \mathrm{i}$ & $-3-4 \mathrm{i}$ & $2.8384151147-0.6200428783 \mathrm{i}$ \\
\hline $6.4+5 \mathrm{i}$ & $-2-2.5 \mathrm{i}$ & $1.2268717607+2.2414666206 \mathrm{i}$ \\
\hline 6.4 & $-1.6+4 \mathrm{i}$ & $0.0956245999+0.7936468285 \mathrm{i}$ \\
\hline
\end{tabular}
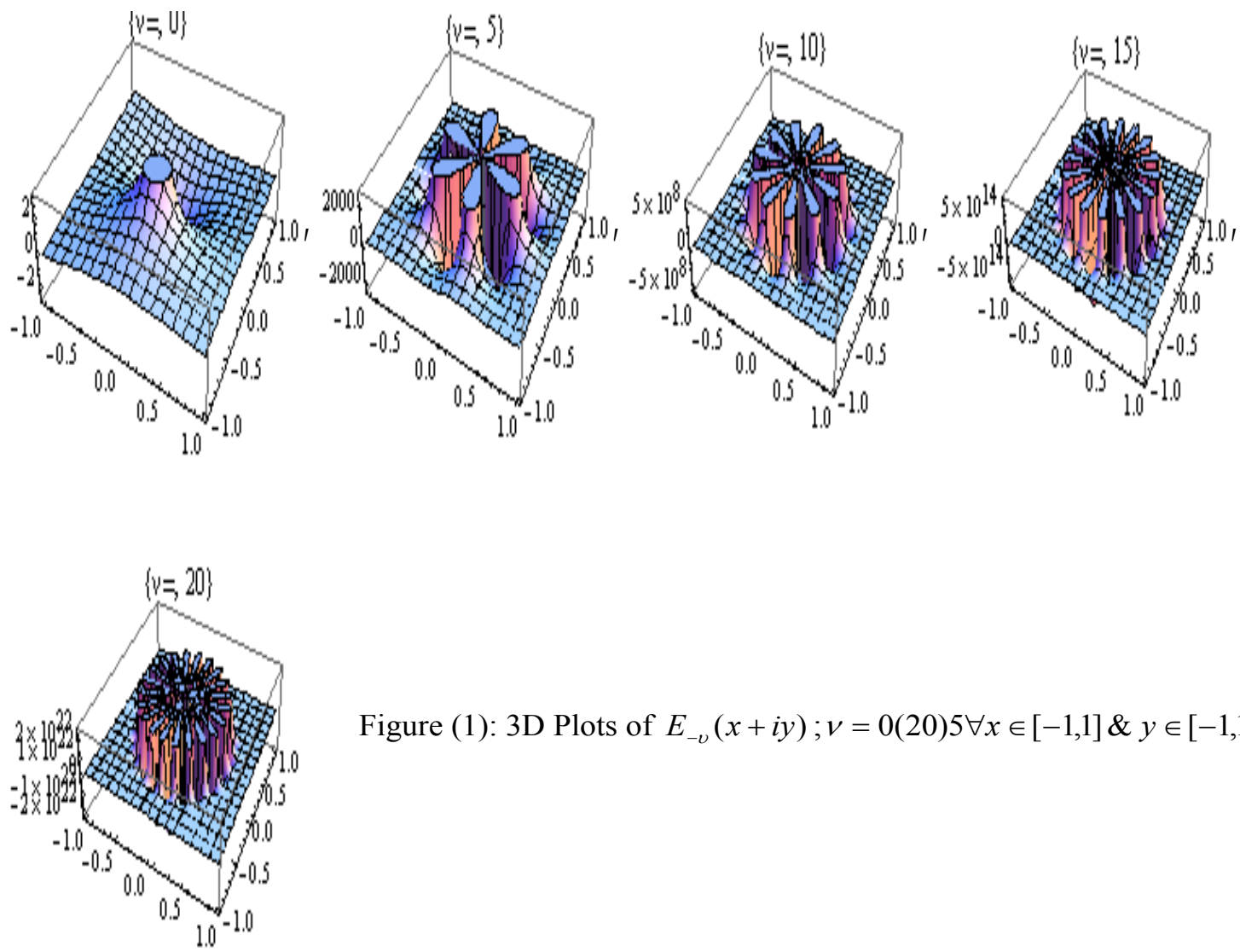

Figure (1): 3D Plots of $E_{-\nu}(x+i y) ; v=0(20) 5 \forall x \in[-1,1] \& y \in[-1,1]$. 


\section{CONCLUSION}

Generally, the continued fraction expansions are more efficient tools for evaluating the classical functions than the more infinite power series. Their convergence is typically faster and more extensive than the series. So, in this paper, an efficient computational algorithm for the exponential integral $E_{v}(z)$ was established for all $v=q_{1}+i q_{2}$ and $z=a+i b$. The algorithm (or Gautschi's algorithm) based on the evaluation of the continued fraction expansion of $E_{v}(z)$. Also, the numerical applications of the algorithm are given in Table (1); and Figure (1) shows 3D representations of $E_{-v}(x+\dot{y})$ for as an example $v=0(2 \varnothing) 5$ for all values of $x$ $\forall x \in[-1,1]$ and $y \forall y \in[-1,1]$.

\section{REFERENCES}

1- George, W.; Hanson; Alexander and Yakovlev, B.: 2002, "Operator Theory for Electromagnetic: An Introduction”, Springer-Verlag, New York.

2- Gautschi, W. and Cahill, W.F.: 1964, "Exponential integral and related functions: Handbook of Mathematical Functions with Formulas, Graphs and Mathematical Tables”, Natl. Bur. Stand. Appl. Math. Ser. 55, Department of Commerce, Washington, DC.

3- Spanier, J. and Oldham, K.B.: 1987, “An Atlas of Functions”, Washington, Hemisphere, Publishing Corporation. Berlin etc., Springer-Verlag, New York.
4- Barcilon, V.: 1990, "Special functions: Handbook of Applied Mathematics", 2. Van Nostrand Reinhold, New York.

5- Michael S. Kluskens: 1999, “A New Algorithm for the Complex Exponential Integral in the Method of Moments", IEEE transactions on antennas and propagation, Vol. 47, No. 5, 803-806.

6- Ala Abu Alkheir and Mohamed Ibnkahla: 2013, "An Accurate Approximation of the Exponential Integral Function Using a Sum of Exponentials", IEEE communications letters, Vol. 17, No. 7, 1364-1367.

7- Abramowitz, M. and Stegun, I.A.: 1972, "Handbook of Mathematical Functions", Dover Publications, Inc., New York.

8- Gautschi, W.: 1967, “Computational Aspects of Threeterm Recurrence Relations", SIAM Review, Vol. 9, No. 1, January.

9- Sharaf, M.A.: 2006, "Computations of the Cosmic Distance Equation”. Appl. Math. Comput. 174, 12691278.

10- Sharaf, M.A.; Almleaky, Y.M; Malawi, A.A.; Goharji, A.A. and Basurah, H.M.: 2004, "Symbolic Analytical Expressions of the Physical Characteristics for N-Dimensional Radially Symmetrical Isothermal Models", AJSE, King Fahd, Univ., Vol. 29. No. 1A, 67-82.

11- Sharaf, M.A. and Banajh, M.A.: 2001, "Continued Fraction Evaluation of the Stumpff Functions of Space Dynamics", Sci. J. Fac., Minufiya Univ., Vol. XV, 267-282.

12- Sharaf, M.A. and Najmuldeen, S.A.: 2001, "Continued Fraction Evaluation of the Normal Distribution Function”, Sci. J. Fac., Minufiya Univ., Vol. XV, 311-324. 